International Journal of Modern Physics E

(C) World Scientific Publishing Company

\title{
DETERMINING THE DENSITY DEPENDENCE OF THE NUCLEAR SYMMETRY ENERGY USING HEAVY-ION REACTIONS
}

\author{
LIE-WEN CHEN \\ Institute of Theoretical Physics, Shanghai Jiao Tong University, \\ Shanghai 200240, China \\ lwchen@sjtu.edu.cn \\ CHE MING KO \\ Cyclotron Institute and Physics Department, Texas A\&M University, \\ College Station, Texas 77843-3366, USA \\ ko@comp.tamu.edu \\ BAO-AN LI \\ Department of Physics, Texas A\&SM University-Commerce, \\ Commerce, Texas 75429, USA \\ Bao-An_Li@TAMU-Commerce.edu \\ GAO-CHAN YONG \\ Institute of Modern Physics, Chinese Academy of Science, Lanzhou 730000, China \\ yonggaochan@impcas.ac.cn \\ Received (received date) \\ Revised (revised date)
}

\begin{abstract}
We review recent progress in the determination of the subsaturation density behavior of the nuclear symmetry energy from heavy-ion collisions as well as the theoretical progress in probing the high density behavior of the symmetry energy in heavy-ion reactions induced by high energy radioactive beams. We further discuss the implications of these results for the nuclear effective interactions and the neutron skin thickness of heavy nuclei.
\end{abstract}

\section{Introduction}

One of the most important properties of a nuclear matter is its equation of state (EOS). For symmetric nuclear matter with equal numbers of protons and neutrons, its EOS has been relatively well determined from the study of the nuclear giant monopole resonances (GMR) 1 as well as the measurements of collective flows 2 and subthreshold kaon production 3 in nucleus-nucleus collisions. On the other hand, our knowledge on the EOS of the isospin asymmetric nuclear matter with unequal numbers of protons and neutrons, especially the part related to the nuclear symme- 
try energy, is still largely uncertain $2|4| 5|6| 7|8| 9 \mid 10$. Although the nuclear symmetry energy at normal nuclear matter density is known to be around $30 \mathrm{MeV}$ from the empirical liquid-drop mass formula 11112 , its values at other densities, particularly at supra-normal densities, are poorly known 45. Advances in radioactive nuclear beam facilities provide, however, the possibility to pin down the density dependence of the nuclear symmetry energy in heavy ion collisions induced by these nuclei 4|5|9|13|14|15|16|17|18|19|20|21|22|23|24|25|26|27|28|29|30|31|32|33|34|35|36|37|38|39|40|41|42|43|

In the present talk, we review recent progress in extracting the information on the subsaturation density behavior of the nuclear symmetry energy from heavy-ion collisions, especially from the analysis of isospin diffusion data 44|45]46. Furthermore, we discuss the implications the constrained symmetry energy has for the nuclear effective interactions and the neutron skin thickness of heavy nuclei. In addition, we also review the theoretical progress on probing the high density behavior of the symmetry energy in heavy-ion reactions induced by high energy radioactive beams.

\section{The nuclear symmetry energy}

The EOS of isospin asymmetric nuclear matter, given by its binding energy per nucleon, can be generally written as

$$
E(\rho, \alpha)=E(\rho, \alpha=0)+E_{\mathrm{sym}}(\rho) \alpha^{2}+O\left(\alpha^{4}\right),
$$

where $\rho=\rho_{n}+\rho_{p}$ is the baryon density with $\rho_{n}$ and $\rho_{p}$ denoting the neutron and proton densities, respectively; $\alpha=\left(\rho_{n}-\rho_{p}\right) /\left(\rho_{p}+\rho_{n}\right)$ is the isospin asymmetry; $E(\rho, \alpha=0)$ is the binding energy per nucleon in symmetric nuclear matter, and

$$
E_{\text {sym }}(\rho)=\left.\frac{1}{2} \frac{\partial^{2} E(\rho, \alpha)}{\partial \alpha^{2}}\right|_{\alpha=0}
$$

is the nuclear symmetry energy. Neglecting contributions from higher-order terms in Eq. (1) leads to the well-known empirical parabolic law for the EOS of asymmetric nuclear matter. For nuclear matter at moderate densities, this parabolic law has been verified in results from all many-body theories. As a good approximation, the symmetry energy can thus be extracted from the binding energy difference between pure neutron matter and symmetric nuclear matter, i.e., $E_{\mathrm{sym}}(\rho) \approx E(\rho, \alpha=1)-$ $E(\rho, \alpha=0)$. It should be mentioned that possible presence of higher-order terms in $\alpha$ at supra-normal densities could significantly modify the proton fraction in $\beta$-equilibrium neutron-star matter and thus the critical density for the direct Urca process that is responsible for faster cooling of neutron stars $47 / 48$.

Around the nuclear matter saturation density $\rho_{0}$, the nuclear symmetry energy $E_{\text {sym }}(\rho)$ can be expanded to second-order in density as

$$
E_{\mathrm{sym}}(\rho)=E_{\mathrm{sym}}\left(\rho_{0}\right)+\frac{L}{3}\left(\frac{\rho-\rho_{0}}{\rho_{0}}\right)+\frac{K_{\mathrm{sym}}}{18}\left(\frac{\rho-\rho_{0}}{\rho_{0}}\right)^{2},
$$


where $L$ and $K_{\text {sym }}$ are the slope and curvature parameters of the nuclear symmetry energy at $\rho_{0}$, i.e.,

$$
L=\left.3 \rho_{0} \frac{\partial E_{\text {sym }}(\rho)}{\partial \rho}\right|_{\rho=\rho_{0}}, K_{\text {sym }}=\left.9 \rho_{0}^{2} \frac{\partial^{2} E_{\text {sym }}(\rho)}{\partial^{2} \rho}\right|_{\rho=\rho_{0}} .
$$

The $L$ and $K_{\text {sym }}$ characterize the density dependence of the nuclear symmetry energy around normal nuclear matter density, and thus carry important information on the properties of nuclear symmetry energy at both high and low densities. In particular, the slope parameter $L$ has been found to correlate linearly with the neutron-skin thickness of heavy nuclei and thus can in principle be determined from measured thickness of the neutron skin of such nuclei 7/49|50|51|52|53|54|55. Unfortunately, because of the large uncertainties in the experimental measurements, no reliable information on the slope parameter of the nuclear symmetry energy has so far been obtained in this way.

At the saturation density and around $\alpha=0$, the isobaric incompressibility of asymmetric nuclear matter can also be expressed to second-order in $\alpha$ as 56157

$$
K(\alpha) \approx K_{0}+K_{\text {asy }} \alpha^{2},
$$

where $K_{0}$ is the incompressibility of symmetric nuclear matter at the saturation density and the isospin-dependent part $K_{\text {asy }} \approx K_{\text {sym }}-6 L 18$ characterizes the density dependence of the nuclear symmetry energy. Information on $K_{\text {asy }}$ can in principle be extracted experimentally by measuring the GMR in neutron-rich nuclei.

\section{Constraining the subsaturation density behavior of the symmetry energy from isospin diffusion data}

The symmetry energy is known to affect the isospin diffusion in intermediate-energy heavy ion collisions 29. Experimentally, the degree of isospin diffusion between the projectile nucleus $A$ and the target nucleus $B$ can be studied via $44 \mid 58$

$$
R_{i}=\frac{2 X^{A+B}-X^{A+A}-X^{B+B}}{X^{A+A}-X^{B+B}},
$$

where $X$ is any isospin-sensitive observable. By construction, the value of $R_{i}$ is $1(-1)$ for symmetric $A+A(B+B)$ reaction. For asymmetric reactions, the value of $R_{i}$ decreases from 1 to about zero as the colliding nuclei approaches isospin equilibrium. In the NSCL/MSU experiments with $A={ }^{124} \mathrm{Sn}$ and $B={ }^{112} \mathrm{Sn}$ at a beam energy of $50 \mathrm{MeV} /$ nucleon and an impact parameter about $6 \mathrm{fm}$, the isospin asymmetry of the projectile-like residue was used as the isospin tracer $X \underline{44}$. Using an isospin- and momentum-dependent IBUU04 transport model with experimental free-space or in-medium nucleon-nucleon (NN) cross sections, the dependence of $R_{i}$ on the nuclear symmetry energy in these reactions was studied in Refs. $45 \mid 46$ for different isospin- and momentum-dependent MDI interactions 59 Shown in Fig. 1 are the results for the degree of the isospin diffusion $1-R_{i}$ as functions of $K_{\text {asy }}$ (left panel) and $L$ (right panel). The shaded band in Fig. 1 indicates the data 
from NSCL/MSU 44 . For the experimental free-space NN cross sections, comparing theoretical results with experimental data allows us to extract a nuclear symmetry energy of $E_{\mathrm{sym}}(\rho) \approx 31.6\left(\rho / \rho_{0}\right)^{1.05}$, corresponding to the parameter $x=-1$ in the MDI interaction. With the medium-dependent NN cross sections, which are important for isospin-dependent observables 46]60, to explain the isospin diffusion data requires an even softer nuclear symmetry energy of $E_{\text {sym }}(\rho) \approx 31.6\left(\rho / \rho_{0}\right)^{\gamma}$ with $\gamma \approx 0.69-1.0546$. In this case, the extracted value for the slope parameter of the nuclear symmetry energy at saturation density is $L=88 \pm 25 \mathrm{MeV}$ and that for the isospin-dependent part of the isobaric incompressibility of isospin asymmetric nuclear matter is of $K_{\text {asy }}=-500 \pm 50 \mathrm{MeV} 45|46| 55$.
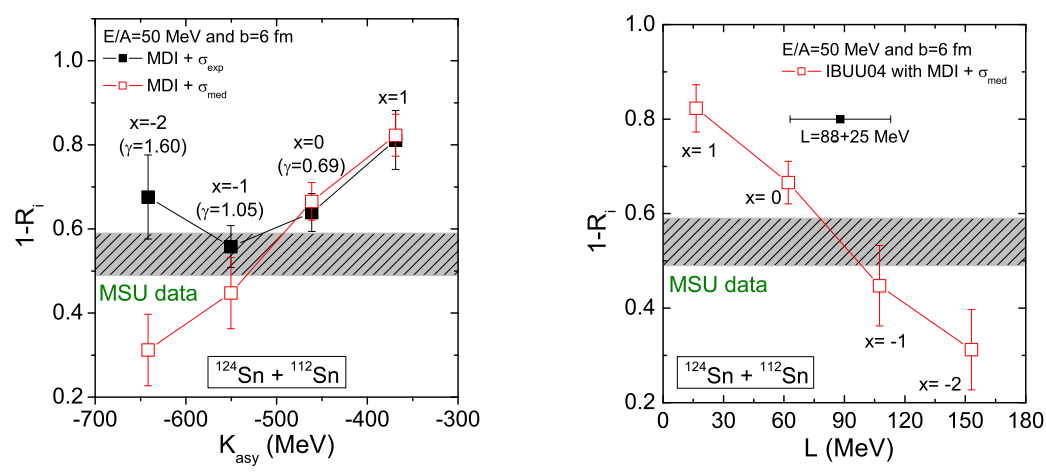

Fig. 1. (Color online) Degree of the isospin diffusion $1-R_{i}$ as functions of $K_{\text {asy }}$ (left panel, taken from Ref. 46) and $L$ (right panel, taken from Ref. 55 ) using the MDI interaction with $x=-2$, $-1,0$, and 1 .

The above extracted symmetry energy agrees with the symmetry energy $E_{\text {sym }}(\rho)=31.6\left(\rho / \rho_{0}\right)^{0.69}$, corresponding to $L \approx 65 \mathrm{MeV}$ and $K_{\text {asy }} \approx-453 \mathrm{MeV}$, that was recently obtained from the isoscaling analyses of isotope ratios in intermediate energy heavy ion collisions $\underline{61}$. The extracted value of $K_{\text {asy }}=-500 \pm 50 \mathrm{MeV}$ from the isospin diffusion data is also consistent with the value $K_{\text {asy }}=-550 \pm 100$ $\mathrm{MeV}$ obtained from recently measured isotopic dependence of the GMR in even-A Sn isotopes 62 . These empirically extracted values for $L$ and $K_{\text {sym }}$ represent the best current constraints on the nuclear symmetry energy at sub-normal densities.

\section{Constraining effective interactions in the SHF and RMF models}

The non-relativistic Skyrme-Hartree-Fock (SHF) 49|63|64|65|66|67 and relativistic mean-field (RMF) 68|69|70|71/72 models are two extensively used phenomenological approaches for nuclear structure studies 73 . In these models, a number of parameters are adjusted to fit the properties of nuclear matter as well as those of many finite nuclei. Since there are many different parameter sets in the SHF and RMF 
models, it is of interest to see to what extend these parameter sets are consistent with the constrained symmetry energy from heavy-ion collisions.
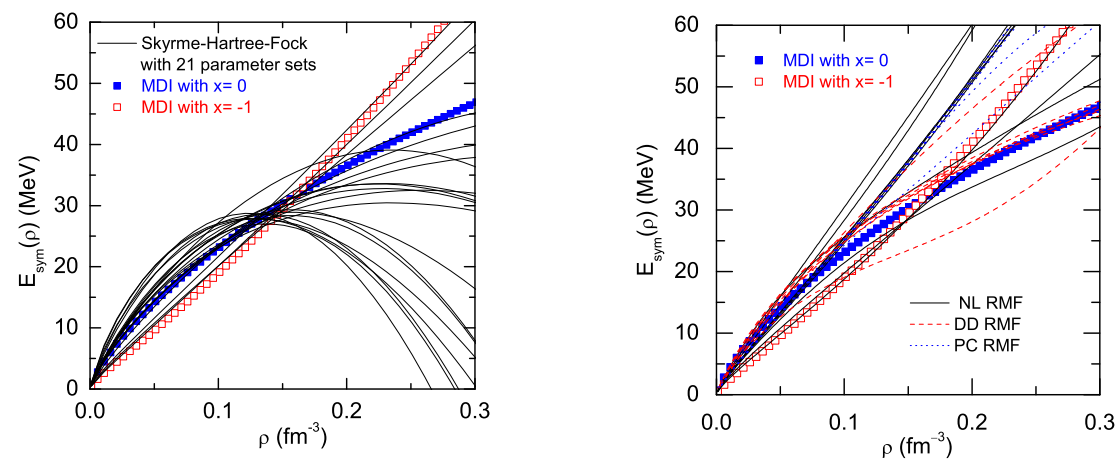

Fig. 2. (Color online) Density dependence of $E_{\text {sym }}(\rho)$ for 21 sets of Skyrme interaction parameters (left panel, taken from Ref. 55 ) and for 23 parameter sets in the nonlinear, density-dependent, and point-coupling RMF models (right panel, taken from Ref. 90 ).

Fig. 2 displays the density dependence of $E_{\mathrm{sym}}(\rho)$ for 21 sets of Skyrme interaction parameters 55 , i.e., SKM, SKM*, RATP, SI, SII, SIII, SIV, SV, SVI, E, E ${ }_{\sigma}$, $\mathrm{G}_{\sigma}, \mathrm{R}_{\sigma}, \mathrm{Z}, \mathrm{Z}_{\sigma}, \mathrm{Z}_{\sigma}^{*}, \mathrm{~T}, \mathrm{~T} 3$, SkX, SkXce, and SkXm (left panel), as well as for 23 parameter sets in the nonlinear (i.e., NL1 74 , NL2 74 , NL3 $\frac{75]}{\text {, NL-SH }}{ }^{76}$, TM1 77 , PK1 18 , FSU-Gold 179 , HA 80 , NL $\rho$ 81, NL $\rho \delta$ 81, density-dependent (i.e., TW99 82, DD-ME1 83, DD-ME2 84, PKDD 78, DD 85, DD-F 86, and DDRH-corr 87),

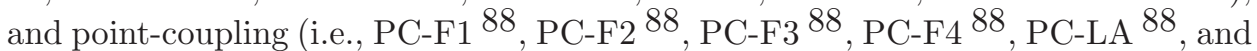
FKVW 89.) RMF models 90 (right panel). Values of the parameters in the Skyrme interactions can be found in Refs. 63|64|65. For comparison, we also show in Fig. 2 results from the MDI interactions with $x=-1$ (open squares) and 0 (solid squares) which give, respectively, the upper and lower bounds for the stiffness of the nuclear symmetry energy from the isospin diffusion data 46 . It is seen that the density dependence of the symmetry energy varies drastically among different interactions and most of the parameter sets give symmetry energies that are inconsistent with the extracted one except SIV, SV, $\mathrm{G}_{\sigma}$, and $\mathrm{R}_{\sigma}$ among the Skyrme parameters 55 and TM1, NL $\rho, \mathrm{NL} \rho \delta, \mathrm{PKDD}$, and FKVW among the RMF parameter sets 90 .

\section{Predictions on the neutron skin thickness of heavy nuclei}

Also affected by the density dependence of nuclear symmetry energy is the neutron skin thickness $S$ of a nucleus, which is defined as the difference between the rootmean-square radii of neutron distribution and proton distribution. In particular, the skin thickness of a nucleus is sensitive to the slope parameter $L$ of the nuclear symmetry energy at normal nuclear matter density 7/49/50|51/52/53/45]. Using above 
21 sets of Skyrme interaction parameters, the neutron skin thickness of several nuclei has been studied and it is found that for heavy nuclei there exists a strong linear correlation between $S$ and $L$. For ${ }^{208} \mathrm{~Pb}$, the linear correlation between $S$ and $L$ is given by the following expression 45 .

$$
\begin{aligned}
S\left({ }^{208} \mathrm{~Pb}\right) & =(0.1066 \pm 0.0019)+\left(0.00133 \pm 3.76 \times 10^{-5}\right) \times L, \\
L & =(-78.5 \pm 3.2)+(740.4 \pm 20.9) \times S\left({ }^{208} \mathrm{~Pb}\right),
\end{aligned}
$$

where the units of $L$ and $S$ are $\mathrm{MeV}$ and fm, respectively. For ${ }^{132} \mathrm{Sn}$ and ${ }^{124} \mathrm{Sn}$, the corresponding expressions are 45

$$
\begin{gathered}
S\left({ }^{132} \mathrm{Sn}\right)=(0.1694 \pm 0.0025)+\left(0.0014 \pm 5.12 \times 10^{-5}\right) \times L, \\
L=(-117.1 \pm 5.4)+(695.1 \pm 25.3) \times S\left({ }^{132} \mathrm{Sn}\right),
\end{gathered}
$$

and

$$
\begin{gathered}
S\left({ }^{124} \mathrm{Sn}\right)=(0.1255 \pm 0.0020)+\left(0.0011 \pm 4.05 \times 10^{-5}\right) \times L, \\
L=(-110.1 \pm 5.2)+(882.6 \pm 32.3) \times S\left({ }^{124} \mathrm{Sn}\right),
\end{gathered}
$$

Similar linear relations between $S$ and $L$ are also expected for other heavy nuclei. This is not surprising since the thickness of the neutron skin in heavy nuclei is determined by the pressure difference between neutron and proton matters in a nucleus, which is proportional to the parameter $L$ 7/49|50|51/52|53/45.

The extracted $L$ value from the isospin diffusion data in heavy ion collisions allows us to determine from Eqs. (7), (9), and (11), respectively, a neutron skin thickness of $0.22 \pm 0.04 \mathrm{fm}$ for ${ }^{208} \mathrm{~Pb}, 0.29 \pm 0.04 \mathrm{fm}$ for ${ }^{132} \mathrm{Sn}$, and $0.22 \pm 0.04 \mathrm{fm}$ for ${ }^{124} \mathrm{Sn}$. We note these results are not only in surprisingly good agreement with those obtained from the RMF model using an accurately calibrated relativistic parametrization, which can describe simultaneously the ground state properties of finite nuclei and their monopole and dipole resonances 79 , but also consistent with the experimental data $91|92| 93$.

\section{Probing the high density behavior of the nuclear symmetry energy in heavy-ion reactions}

Although significant progress has been made in the determination of the density dependence of the nuclear symmetry energy at sub-normal densities, the high density behavior of the the nuclear symmetry energy is still poorly known. Fortunately, heavy-ion reactions, especially those induced by high energy radioactive beams to be available at high energy radioactive beam facilities, provide a unique opportunity to pin down the high density behavior of the symmetry energy. In this section, we illustrate via transport model simulations several experimental observables which are sensitive to the high density behavior of the symmetry energy.

Most observables proposed so far for studying the density dependence of the nuclear symmetry energy employ differences or ratios of isospin multiplets of baryons 
and mesons as well as mirror nuclei, such as the neutron/proton ratio of emitted nucleons 13 , neutron-proton differential flow 21, neutron-proton correlation function $23, t /{ }^{3} \mathrm{He} 24\left|34, \pi^{-} / \pi^{+} 22\right| 32|33| 36, \Sigma^{-} / \Sigma^{+} 35$ and $K^{0} / K^{+}$ratios 94|95|96, etc. However, some of these observables, especially those involving neutrons, pose great challenges in experimental measurements. The measurement of neutrons, particularly the low energy ones, always suffers from low detection efficiencies even for the most advanced neutron detectors. Therefore, observables involving neutrons normally have large systematic errors. Moreover, for essentially all these observables, the Coulomb force on charged particles plays an important role and sometimes competes strongly with the symmetry potential. It is thus very desirable to find experimental observables which can reduce the influence of both the Coulomb force and the systematic errors associated with the measurement of neutrons. A possible candidate for such an observable is the double ratios of emitted particles taken from two reaction systems using four isotopes of same element, such as the neutron/proton ratio in the neutron-rich system over that in the more symmetric system, as recently done by Famiano et al. 97 . Theoretically, the systematic errors associated with transport model calculations, which are mostly related to the uncertainties in the in-medium NN cross sections, techniques of treating collisions, sizes of the lattices in calculating the phase space distributions, techniques in handling the Pauli blocking, etc., are also expected to be reduced for relative observables that involve double ratios from two similar reaction systems.
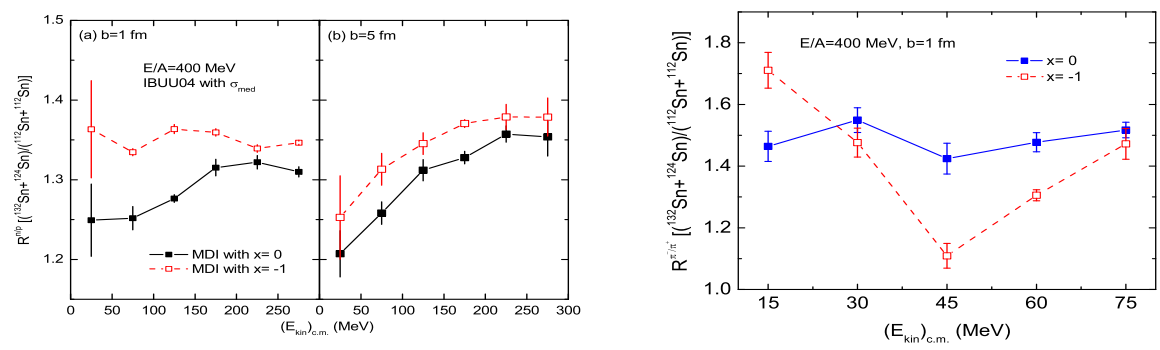

Fig. 3. (Color online) Double ratio for neutron/proton (left panels, taken from Ref. 40 ) and $\pi^{-} / \pi^{+}$ (right panel, taken from Ref. 41) in ${ }^{132} \mathrm{Sn}+{ }^{124} \mathrm{Sn}$ and ${ }^{112} \mathrm{Sn}+{ }^{112} \mathrm{Sn}$ reactions at $400 \mathrm{MeV} / \mathrm{nucleon}$.

Both the double neutron/proton ratio 40 and the double $\pi^{-} / \pi^{+}$ratio 41 in ${ }^{132} \mathrm{Sn}+{ }^{124} \mathrm{Sn}$ and ${ }^{112} \mathrm{Sn}+{ }^{112} \mathrm{Sn}$ reactions at $400 \mathrm{MeV} /$ nucleon have been studied recently in the IBUU04 model in order to demonstrate the effect of symmetry energy at high density, and the results are shown in Fig. 3. It is seen that these ratios have about the same sensitivity to the density dependence of symmetry energy as the corresponding single ratio in the respective neutron-rich system. Also, the double neutron-proton differential flow ${ }^{42}$ in ${ }^{132} \mathrm{Sn}+{ }^{124} \mathrm{Sn}$ and ${ }^{112} \mathrm{Sn}+{ }^{112} \mathrm{Sn}$ 


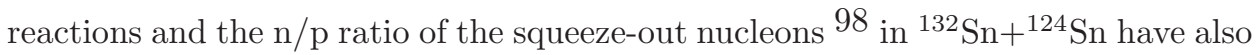
recently been studied in the IBUU04 model. As shown in Fig. 4 , both are indeed sensitive to the symmetry energy. In particular, compared to other potential probes, the $\mathrm{n} / \mathrm{p}$ ratio of squeeze-out nucleons carries more direct information about the symmetry energy at high densities. The sensitivity to the high density behavior of the nuclear symmetry energy observed in the $\mathrm{n} / \mathrm{p}$ ratio of squeeze-out nucleons with high transverse momenta is probably the highest found so far among all observables studied within the same transport model.
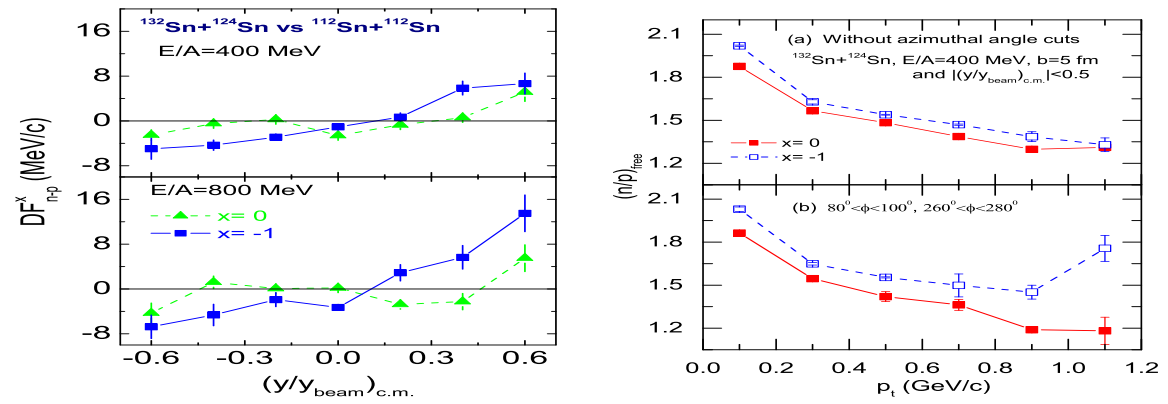

Fig. 4. (Color online) Left panels: Rapidity dependence of the double $\mathrm{n}-\mathrm{p}$ differential transverse flow in reactions of $\mathrm{Sn}+\mathrm{Sn}$ isotopes at 400 and $800 \mathrm{MeV} /$ nucleon with $b=5 \mathrm{fm}$ (Taken from Ref. $\frac{42}{3}$ ). Right panels: Transverse momentum dependence of the ratio of midrapidity neutrons to protons emitted in the reaction of ${ }^{132} \mathrm{Sn}+{ }^{124} \mathrm{Sn}$ at $400 \mathrm{MeV} /$ nucleon and $b=5 \mathrm{fm}$, with (lower window) and without (upper window) an azimuthal angle cut (Taken from Ref. 98, ).

Since the proposal of Aichelin and Ko 99 that the kaon yield in heavy ion collisions at energies that are below the threshold for kaon production in a nucleonnucleon collision in free space may be a sensitive probe of the EOS of nuclear matter at high densities, a lot of works have been done both theoretically and experimentally on this problem $3 / 100|101| 102|103| 104$. As the kaon is an iso-doublet meson with the quark content of $d \bar{s}$ for $K^{0}$ and $u \bar{s}$ for $K^{+}$, the $K^{0} / K^{+}$ratio provides a potentially good probe of the nuclear symmetry energy as the $n / p$ and $\pi^{-} / \pi^{+}$ratios. The $K^{0} / K^{+}$ratio is expected to be particularly sensitive to the high density behavior of nuclear symmetry energy as kaons are produced mainly from the high density region during the early stage of the reaction and thus suffer negligible absorption effects. The symmetry energy effect on the $K^{0} / K^{+}$ratio in heavy-ion collisions has recently been investigated using the UrQMD 94 and RBUU models $95 \mid 96$. The results from the RBUU model 95 , shown in the left panel of Fig. 5 indicate that at beam energies below and around the kinematical threshold of kaon production, the $K^{0} / K^{+}$inclusive yield ratio is more sensitive to the symmetry energy than the $\pi^{-} / \pi^{+}$. Subthreshold kaon production thus could provide a promising tool to extract information on the density dependence of the nuclear symmetry energy.

Experimentally, the FOPI collaboration at GSI-Darmstadt has recently reported 

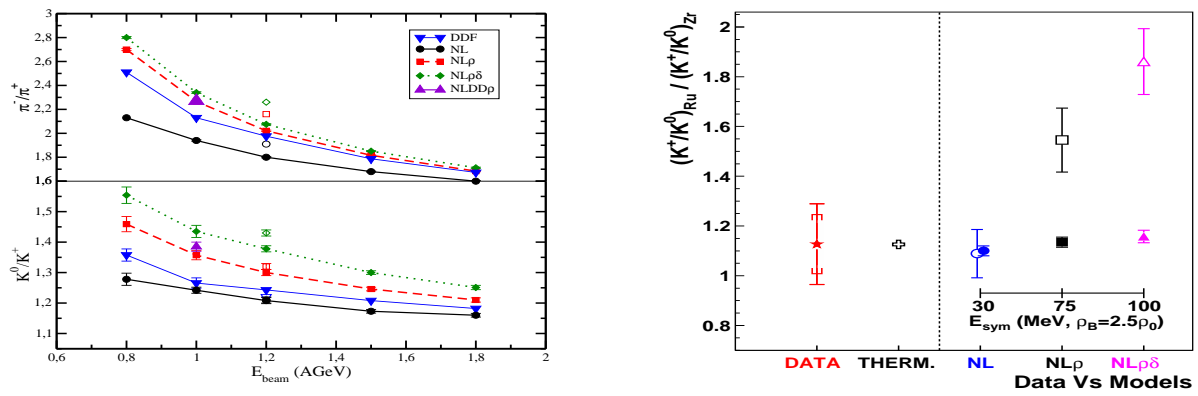

Fig. 5. (Color online) Left panel: $\pi^{-} / \pi^{+}$(upper) and $K^{+} / K^{0}$ (lower) ratios as a function of the incident energy for central Au+Au collisions with the RBUU model (Taken from Ref. 95). Right panel: Experimental ratio $\left(K^{+} / K^{0}\right)_{R u} /\left(K^{+} / K^{0}\right)_{Z_{r}}$ (star) and theoretical predictions of the thermal model (cross) and the transport model with nuclear matter (open symbols) and realistic (filled symbols) collision scenarios (Taken from Ref. 105).

the results on $K^{+}$and $K^{0}$ meson production in ${ }_{44}^{96} \mathrm{Ru}+{ }_{44}^{96} \mathrm{Ru}$ and ${ }_{40}^{96} \mathrm{Zr}+{ }_{40}^{96} \mathrm{Zr}$ collisions at a beam kinetic energy of $1.528 \mathrm{~A} \mathrm{GeV}$ 105. The measured double ratio $\left(K^{+} / K^{0}\right)_{R u} /\left(K^{+} / K^{0}\right)_{Z r}$ is compared in the right panel of Fig. 5 to the predictions from both the thermal model and the RBUU transport model using two different collision scenarios and under different assumptions on the stiffness of the symmetry energy. The experimental data show a good agreement with the thermal model prediction and also with that of the transport model using the collision scenario of an infinite nuclear matter with a soft symmetry energy. Although more realistic transport simulations of the collisions show a similar agreement with the data, they exhibit a rather weak sensitivity to the symmetry energy. Due to the complexity of subthreshold kaon production in heavy-ion collisions $104 \mid 3$, further experimental and theoretical studies are needed to extract useful information on the high density behavior of the nuclear symmetry energy from subthreshold kaon production in heavy-ion collisions induced by neutron-rich nuclei.

\section{Summary}

Heavy-ion reactions induced by neutron-rich nuclei provide a unique means to investigate the equation of state of isospin-asymmetric nuclear matter, especially the density dependence of the nuclear symmetry energy. We have reviewed the recent progress in extracting the information on the subsaturation density behavior of the nuclear symmetry energy from heavy-ion collisions. From the recent analysis of the isospin diffusion data in heavy-ion collisions using an isospin- and momentum-dependent transport model with in-medium $\mathrm{NN}$ cross sections, a value of $L=88 \pm 25 \mathrm{MeV}$ for the slope parameter of the nuclear symmetry energy at saturation density and a value of $K_{\text {asy }}=-500 \pm 50 \mathrm{MeV}$ for the isospin-dependent part of the isobaric incompressibility of isospin asymmetric nuclear matter have been extracted. The extracted symmetry energy agrees with the symmetry energy 
$E_{\text {sym }}(\rho)=31.6\left(\rho / \rho_{0}\right)^{0.69}$, corresponding to $L \approx 65 \mathrm{MeV}$ and $K_{\text {asy }} \approx-453 \mathrm{MeV}$, that was recently obtained from the isoscaling analyses of isotope ratios in intermediate energy heavy ion collisions 61 . The extracted value of $K_{\text {asy }}=-500 \pm 50 \mathrm{MeV}$ from the isospin diffusion data is also consistent with the value $K_{\text {asy }}=-550 \pm 100$ $\mathrm{MeV}$ obtained from recently measured isotopic dependence of the GMR in even-A Sn isotopes 62 . These empirically extracted values for $L$ and $K_{\text {sym }}$ represent the best current constraints on the nuclear symmetry energy at sub-normal densities. This in turn imposes strong constraints on the parameters in both Skyrme and RMF effective interactions. Detailed studies have indicated that only SIV, SV, $\mathrm{G}_{\sigma}$, and $\mathrm{R}_{\sigma}$ among the Skyrme parameters and only TM1, NL $\rho, \mathrm{NL} \rho \delta$, PKDD, and FKVW among the RMF parameter sets have symmetry energies that are consistent with the extracted one. Furthermore, the extracted $L$ value from the isospin diffusion data has led to predicted neutron skin thickness of $0.22 \pm 0.04 \mathrm{fm}$ for ${ }^{208} \mathrm{~Pb}, 0.29 \pm 0.04 \mathrm{fm}$ for ${ }^{132} \mathrm{Sn}$, and $0.22 \pm 0.04 \mathrm{fm}$ for ${ }^{124} \mathrm{Sn}$.

We have also reviewed recent theoretical progress in identifying the observables in heavy-ion collisions induced by high energy radioactive nuclei that are sensitive to the high density behavior of the symmetry energy. Many potentially sensitive probes have been found, and they include the $\pi^{-} / \pi^{+}$ratio, isospin fractionation, $\mathrm{n}-\mathrm{p}$ differential flow, double $\mathrm{n} / \mathrm{p}$ and $\pi^{-} / \pi^{+}$ratios, double $\mathrm{n}-\mathrm{p}$ differential transverse flow as well as the $K^{0} / K^{+}$and $\Sigma^{-} / \Sigma^{+}$ratios. Studying these observable in future experiments at high energy radioactive beam facilities is expected to provide significant constraints on the behavior of the symmetry energy at supra-normal densities.

\section{Acknowledgments}

This work was supported in part by the National Natural Science Foundation of China under Grant Nos. 10575071 and 10675082, MOE of China under project NCET-05-0392, Shanghai Rising-Star Program under Grant No. 06QA14024, the SRF for ROCS, SEM of China, the China Major State Basic Research Development Program under Contract No. 2007CB815004, the US National Science Foundation under Grant Nos. PHY-0457265 and PHY-0652548, the Welch Foundation under Grant No. A-1358, and the Research Corporation under Award No. 7123.

\section{References}

1. D.H. Youngblood, H.L. Clark, and Y.W. Lui, Phys. Rev. Lett. 82 (1999) 691.

2. P. Danielewicz, R. Lacey, and W.G. Lynch, Science 298 (2002) 1592.

3. C. Fuchs, Prog. Part. Nucl. Phys. 56 (2006) 1.

4. B.A. Li, C.M. Ko, and W. Bauer, Int. Jour. Mod. Phys. E 7 (1998) 147.

5. Isospin Physics in Heavy-Ion Collisions at Intermediate Energies, Eds. Bao-An Li and W. Udo Schröder (Nova Science Publishers, Inc, New York, 2001).

6. I. Bombaci, in Ref. [2], p.35.

7. A.E.L. Dieperink et al., Phys. Rev. C 68 (2003) 064307.

8. J.M. Lattimer and M. Prakash, Science 304 (2004) 536. 
9. V. Baran, M. Colonna, V. Greco, and M. Di Toro, Phys. Rep. 410 (2005) 335.

10. A.W. Steiner, M. Prakash, J.M. Lattimer, and P.J. Ellis, Phys. Rep. 411 (2005) 325.

11. W.D. Myers and W.J. Swiatecki, Nucl. Phys. A81 (1966) 1.

12. K. Pomorski and J. Dudek, Phys. Rev. C 67 (2003) 044316.

13. B.A. Li, C.M. Ko, and Z.Z. Ren, Phys. Rev. Lett. 78 (1997) 1644.

14. B.A. Li and C.M. Ko, Nucl. Phys. A618 (1997) 498.

15. V. Baran et al., Nucl. Phys. A632 (1998) 287.

16. H.S. Xu et al., Phys. Rev. Lett. 85 (2000) 716.

17. W.P. Tan et al., Phys. Rev. C64, (2001) 051901(R).

18. V. Baran et al., Nucl. Phys. A703 (2002) 603.

19. M.B. Tsang et al., Phys. Rev. Lett. 86 (2001) 5023.

20. B.A. Li, A.T. Sustich, and B. Zhang, Phys. Rev. C64 (2001) 054604.

21. B.A. Li, Phys. Rev. Lett. 85 (2000) 4221.

22. B.A. Li, Phys. Rev. Lett. 88 (2002) 192701; Nucl. Phys. A708 (2002) 365.

23. L.W. Chen et al., Phys. Rev. Lett. 90 (2003) 162701; Phys. Rev. C 68 (2003) 014605.

24. L.W. Chen, C.M. Ko, and B.A. Li, Phys. Rev. C C68 (2003) 017601; Nucl. Phys. A729 (2003) 809.

25. A. Ono et al., Phys. Rev. C68 (2003) 051601(R).

26. J.Y. Liu, W.J. Guo, Y.Z. Xing, and H. Liu, Nucl. Phys. A726 (2003) 123.

27. L.W. Chen, C.M. Ko, and B.A. Li, Phys. Rev. C 69 (2004) 054606.

28. B.A. Li et al., Phys. Rev. C 69 (2004) 011603(R); Nucl. Phys. A735 (2004) 563.

29. L. Shi and P. Danielewicz, Phys. Rev. C 68 (2003) 064604.

30. B.A. Li, Phys. Rev. C 69 (2004) 034614.

31. J. Rizzo, M. Colonna, M. Di Toro, and V. Greco, Nucl. Phys. A732 (2004) 202.

32. T. Gaitanos et al., Nucl. Phys. A732 (2004) 24.

33. B.A. Li et al., Phys. Rev. C71 (2005) 014608; ibid C 71 (2005) 044604.

34. Y. Zhang and Z. Li, Phys. Rev. C 71 (2005) 024604.

35. Q. Li, Z. Li, E. Zhao, and R.K. Gupta, Phys. Rev. C 71 (2005) 054907.

36. Q.F. Li et al., Phys. Rev. C 72 (2005) 034613;

37. Y.G. Ma et al., Phys.Rev. C 69 (2004) 064610; ibid C 72 (2005) 064603.

38. W.D. Tian et al., Chin. Phys. Lett. 22 (2005) 306; Phys. Rev. C 76, (2007) 024607.

39. W. Trautmann, arXiv:nucl-ex/0610032.

40. B.A. Li, L.W. Chen, G.C Yong, and W. Zuo, Phys. Lett. B634 (2006) 378.

41. G.C. Yong, B.A. Li, L.W. Chen, and W. Zuo, Phys. Rev. C 73 (2006) 034603.

42. G.C. Yong, B.A. Li, L.W. Chen, Phys. Rev. C 74 (2006) 064617.

43. B.A. Li et al., arXiv:0710.2877 [nucl-th], PRC (2007) in press.

44. M.B. Tsang et al., Phys. Rev. Lett. 92 (2004) 062701.

45. L.W. Chen, C.M. Ko, and B.A. Li, Phys. Rev. Lett. 94 (2005) 032701.

46. B.A. Li and L.W. Chen, Phys. Rev. C72 (2005) 064611.

47. F.S. Zhang and L.W. Chen, Chin. Phys. Lett. 18, 142 (2001).

48. A.W. Steiner, Phys. Rev. C 74 (2006) 045808.

49. B.A. Brown, Phys. Rev. Lett. 85 (2000) 5296.

50. C.J. Horowitz and J. Piekarewicz, Phys. Rev. Lett 86 (2001) 5647; Phys. Rev. C 66 (2002) 055803.

51. S. Typel and B.A. Brown, Phys. Rev. C 64 (2001) 027302.

52. R.J. Furnstahl, Nucl. Phys. A706 (2002) 85.

53. S. Karataglidis, K. Amos, B.A. Brown, and P.K. Deb, Phys. Rev. C65 (2002) 044306.

54. A.W. Steiner and B.A. Li, Phys. Rev. C 72 (2005) 041601.

55. L.W. Chen, C.M. Ko, and B.A. Li, Phys. Rev. C 72 (2005) 064309.

56. M. Prakash and K. S. Bedell, Phys. Rev. C 32 (1985) 1118. 
57. M. Lopez-Quelle et al., Nucl. Phys. A483 (1988) 479.

58. F. Rami et al., Phys. Rev. Lett. 84 (2000) 1120.

59. C.B. Das, S. Das Gupta, C. Gale, and B.A. Li, Phys. Rev. C 67 (2003) 034611.

60. B.A. Li, P. Danielewicz, and W.G. Lynch, Phys. Rev. C 71 (2005) 054603.

61. D. Shetty, S.J. Yennello and G.A. Souliotis, Phys. Rev. C 75 (2007) 034602.

62. T. Li et al., Phys. Rev. Lett. 99 (2007) 162503.

63. M. Brack, C. Guet and H.-B. Hakansson, Phys. Rep. 123 (1985) 275.

64. J. Friedrich and P.-G. Reinhard, Phys. Rev. C 33 (1986) 335.

65. B.A. Brown, Phys. Rev. C 58 (1998) 220.

66. L.W. Chen and F.S. Zhang, High Energy Phys. and Nucl. Phys. 23 (1999) 1197.

67. J.R. Stone et al., Phys. Rev. C 68 (2003) 034324.

68. B.D. Serot and J.D. Walecka, Adv. Nucl. Phys. 16 (1986) 1.

69. P.-G. Reinhard, Rep. Prog. Phys. 52 (1989) 439.

70. P. Ring, Prog. Part. Nucl. Phys. 37 (1996) 193.

71. B.D. Serot and J.D. Walecka, Int. J. Mod. Phys. E 6 (1997) 515.

72. J. Meng et al., Prog. Part. Nucl. Phys. 57 (2006) 470.

73. M. Bender, P.-H. Heenen, P.-G. Reinhard, Rev. Mod. Phys. 75 (2003) 121.

74. S.-J. Lee et al., Phys. Rev. Lett. 57 (1986) 2916.

75. G.A. Lalazissis, J. Konig, P. Ring, Phys. Rev. C 55 (1997) 540.

76. M.M. Sharma, M.A. Nagarajan, P. Ring, Phys. Lett. B312 (1993) 377.

77. Y. Sugahara and H. Toki, Nucl. Phys. A579 (1994) 557.

78. W.H. Long, J. Meng, N. Van Giai, S.G. Zhou, Phys. Rev. C 69 (2004) 034319.

79. B.G. Todd-Rutel and J. Piekarewicz, Phys. Rev. Lett. 95 (2005) 122501.

80. J.K. Bunta and S. Gmuca, Phys. Rev. C 68 (2003) 054318.

81. B. Liu et al., Phys. Rev. C 65 (2002) 045201.

82. S. Typel and H. H. Wolter, Nucl. Phys. A656 (1999) 331.

83. T. Niksic, D. Vretenar, P. Finelli, P. Ring, Phys. Rev. C 66 (2002) 024306.

84. G.A. Lalazissis, T. Niksic, D. Vretenar, and P. Ring, Phys. Rev. C 71 (2005) 024312.

85. S. Typel, Phys.Rev. C 71 (2005) 064301.

86. T. Klahn et al., Phys.Rev. C 74 (2006) 035802.

87. F. Hofmann, C. M.Keil, and H. Lenske, Phys. Rev. C 64 (2001) 034314.

88. T. Bürvenich et al., Phys. Rev. C 65 (2002) 044308.

89. P. Finelli, N. Kaiser, D. Vretenar and W. Weise, Nucl. Phys. A770 (2006) 1.

90. L.W. Chen, C.M. Ko and B.A. Li, arXiv:0709.0900 [nucl-th], PRC, (2007) in press.

91. V.E. Starodubsky and N.M. Hintz, Phys. Rev. C 49 (1994) 2118.

92. B.C. Clark, L.J. Kerr, and S. Hama, Phys. Rev. C 67 (2003) 054605.

93. A. Krasznahorkay et al., Nucl. Phys. A731 (2004) 224.

94. Q. Li et al., J. Phys. G 31 (2005) 1359.

95. G. Ferini, M. Colonna, T. Gaitanos, M. Di Toro, Nucl. Phys. A762 (2005) 147.

96. G. Ferini et al., Phys. Re. Lett. 97 (2006) 202301.

97. M. A. Famiano et al., Phys. Rev. Lett. 97 (2006) 052701.

98. G.C. Yong, B.A. Li, and L.W. Chen, Phys. Lett. B650 (2007) 344.

99. J. Aichelin and C.M. Ko, Phys. Rev. Lett. 55 (1985) 2661.

100. W. Cassing, V. Metag, U. Mosel and K. Niita, Phys. Rep. 188 (1990) 363.

101. C.M. Ko and G.Q. Li, J. Phys. G 22 (1996) 1673.

102. C.M. Ko, V. Koch and G.Q. Li, Ann. Rev. Nucl. Part. Sci. 47 (1997) 505.

103. W. Cassing and E. L. Bratkovskaya, Phys. Rep. 308 (1999) 65.

104. E.E. Kolomeitsev et al., J. Phys. G 31 (2005) S741.

105. X. Lopez et al. (FOPI Collaboration), Phys. Rev. C 75 (2007) 011901(R). 